\title{
Sacrificio de pacientes con COVID-19 grave durante las primeras semanas de la pandemia en Perú: Actitudes y factores asociados
}

\section{Sacrifice of patients with severe COVID-19 during the first weeks of the pandemic in Peru: Attitudes and associated factors}

\author{
https://doi.org/10.52808/bmsa.7e5.61e2.017 \\ Christian R. Mejia ${ }^{1 *}$ \\ https://orcid.org/0000-0002-5940-7281 \\ Katherin Huaroc-Segovia ${ }^{2}$ \\ https://orcid.org/0000-0001-7022-1886 \\ Ivan Chambergo ${ }^{2}$ \\ https://orcid.org/0000-0001-6642-9246 \\ Daniella Vinelli-Arzubiaga ${ }^{3}$ \\ https://orcid.org/0000-0002-8793-2446 \\ Anthony Bautista-Pariona ${ }^{4}$ \\ https://orcid.org/0000-0002-3499-4491 \\ Dennis Arias-Chávez ${ }^{5}$ \\ https://orcid.org/0000-0003-1500-8366 \\ Martin A. Vilela-Estrada ${ }^{6}$ \\ https://orcid.org/0000-0002-1494-952X \\ Victor Serna-Alarcón 6 \\ https://orcid.org/0000-0002-9803-6217
}

Recibido: $11 / 03 / 2021$

Aceptado: 23/06/2021

\section{RESUMEN}

Hubo mucho desconcierto y temor cuando llegó el COVD-19 al país, tanto así que algunos opinaban que se tomen medidas extremas ante los casos confirmados, sin embargo, esto no había sido evaluado. El objetivo de la investigación fue el determinar los factores asociados a estar de acuerdo con sacrificar a las personas que padecían de COVID-19 grave durante las primeras semanas de la pandemia en el Perú. Estudio transversal analítico, que usó una data secundaria, la variable dependiente fue la percepción de estar de acuerdo que si alguien tiene un caso grave de coronavirus debería ser sometido a un sacrificio/eutanasia o similar, para evitar el contagio; esto se cruzó con otras variables influyentes. De los 3331 encuestados, el $8 \%$ (269) estaban muy de acuerdo y el 9\% (297) de acuerdo con que sacrifiquen a las personas con COVID-19 grave. En el análisis multivariado, el estar de acuerdo con el sacrificio se asoció al sexo (las mujeres estaban menos de acuerdo con el sacrificar, RPa: 0,73; IC95\%: 0,62-0,86; p<0,001), también según la orientación religiosa (los ateos estaban más de acuerdo con el sacrificar, RPa: 1,40; IC95\%: 1,16-1,69; p<0,001), y a los puntajes que obtuvieron en los test's de fatalismo (RPa: 1,09; IC95\%: 1,07-1,11; $\mathrm{p}<0,001$ ), nivel de conocimientos (RPa: 0,85; IC95\%: 0,81-0,88; $<<0,001$ ) y la percepción de miedo o exageración por parte de los medios de comunicación (RPa: 1,02; IC95\%: 1,01-1,03; p <0,001). Un importante porcentaje estuvo de acuerdo con sacrificar a los enfermos, estando asociado al miedo, fatalismo y bajo nivel de conocimientos.

Palabras clave: Sacrificio, conocimiento, medios de comunicación, miedo, Perú.

\section{ABSTRACT}

There was a lot of confusion and fear when COVD-19 arrived to the country, so much so that some were of the opinion that extreme measures should be taken for confirmed cases, however, this had not been evaluated. The objective of the research was to determine the factors associated with agreement to sacrifice people with severe COVD-19 during the first weeks of the pandemic in Peru. Cross-sectional analytical study, using secondary data, the dependent variable was the perception of agreement that if someone has a severe case of coronavirus they should be sacrificed/euthanized or similar, to avoid contagion; this was crossed with other influential variables. Of the 3331 respondents, 8\% (269) strongly agreed and 9\% (297) agreed that people with severe COVID-19 should be euthanized. In multivariate analysis, agreeing with culling was associated with sex (women were less in agreement with sacrificing, aPR: 0.73; 95\% CI: 0.62-0.86; $p<0.001)$, also according to religious orientation (atheists were more in agreement with culling, aPR: 1.40; 95\% CI: 1.16-1.69; $p<0.001)$, and to the scores they obtained in the fatalism test's (aPR: 1.09; CI95\%: $1.07-1.11 ; p<0.001)$, level of knowledge (aPR: 0.85; C195\%: 0.81-0.88; $p<0.001)$ and the perception of fear or exaggeration by the media (aPR: 1.02; CI95\%: 1.01-1.03; p $<0.001)$. A significant percentage agreed with sacrificing the sick people, being this associated with fear, fatalism and a low level of knowledge.

Key words: Sacrifice, knowledge, social media, fear, Peru.

1. Centro de Investigación en Medicina Traslacional. Universidad Norbert Wiener. Lima, Perú 2. Universidad Continental. Huancayo, Perú. 


\section{Introducción}

Antes de la llegada de la pandemia del COVID-19 existían algunas enfermedades que causaban mucho miedo al enterarse que las padecían. Por ejemplo, algunas personas al saber que se encontraban infectadas por el Virus de la Inmunodeficiencia Humana (VIH) tomaban decisiones fatales, provocándose intencionalmente la muerte; esto bajo la idea de que culminar con su sufrimiento, evitar el estigma social, el sentimiento de soledad y las complicaciones a las que se enfrentaban, entre otras (Alderete-Aguilar et al., 2017; Carrieri et al., 2017). Esto también se ha evidenciado en pacientes con cáncer de páncreas, pues es conocido que esta enfermedad tiene un pronóstico sombrío, causando mucho dolor en las etapas finales (Du et al., 2020). Lo que en su conjunto nos muestra que algunos adoptaban una posición extrema, pero que se considera el "mal menor"; reflejando el miedo al sufrimiento, a la agonía propia o de nuestros seres queridos, al ser señalados, entre otras muchas que se iniciaban con una enfermedad que no tiene una cura conocida; siendo un dilema ético el sacrificio de pacientes terminales o de aquellos que sufren enfermedades incurables, que generan mucho sufrimiento y eventualmente conducen a la muerte (Dubón-Peniche \& Bustamante-Leija, 2020). Si bien el paciente tiene el derecho de decidir sobre su propio cuerpo, según el principio de autonomía, aún existen otras consideraciones éticas médicas y familiares que deben ser tomadas en cuenta al momento de hablar de eutanasia (Rodriguez Casas, 2001; Torres Lio-Coo et al., 2009).

Con la llegada de la COVID-19, una enfermedad potencialmente mortal y que no tuvo un tratamiento adecuado durante el primer año, se observó que algunas personas vieron afectada su salud mental y tuvieron actitudes extremas (Asmundson \& Taylor, 2020; Pirkis et al., 2021). Además, algunos opinaban que se debía ser muy restrictivo con las personas, en los aspectos relacionados al aislamiento social obligatorio; otros planteaban sanciones muy drásticas con aquellos que incumplan las normas de bioseguridad (Abdoli, 2020; White \& Fradella, 2020). En este contexto también llegaron noticias extremas desde Corea del Norte, donde se especuló que "se dejaba morir" a aquellas personas que presentaban esta enfermedad (Infobae, 2020); sin embargo, esto no llegó a ser confirmado por el régimen que gobierna el país, ya que, como bien se sabe, las medidas políticas impiden que se pueda obtener información verídica y de primera mano.

Todo lo ya mencionado nos muestra la importancia de caracterizar algunas reacciones, percepciones o formas extremas de pensar que se pudieron tener al enfrentarse a esta pandemia. Si bien en el Perú se realizaron estudios para evaluar el fatalismo de la población peruana ante la pandemia de COVID-19 (Mejia et al., 2020 a), no existen estudios que evalúen las actitudes de la población ante aquellas personas que se contagiaron de COVID-19 durante la fase de instauración de la pandemia; por ello, el objetivo de esta investigación fue el determinar los factores asociados a estar de acuerdo con sacrificar a las personas que padecían de COVID-19 grave durante las primeras semanas de la pandemia en el Perú.

\section{Materiales y Métodos}

Estudio transversal analítico. Que se basó en el análisis de una pregunta que se aplicó a través de una encuesta virtual a varias ciudades del Perú; todo durante los primeros días de la cuarentena generada por la primera ola del COVID-19 en el país (segunda quincena de marzo y primera quincena de abril). La población que se tomó en cuenta para este estudio provenía en su mayoría de las clases económicas B, C y D. Se incluyó en la investigación a personas mayores de 18 años, que residían en alguno de los departamentos del Perú durante la primera ola y a aquellos que aceptaron participar de manera voluntaria. Se excluyó a las personas que no respondieron a la pregunta principal de la encuesta o que dieron respuestas incoherentes (menor del $3 \%$ de excusiones de la base total).

Todo esto se obtuvo a partir de un muestreo de tipo no aleatorio. El tipo de investigación generó que se tuviese que calcular la potencia estadística de los cruces, ya que, todo esto fue producto de un análisis secundario de una investigación que mostró las medidas que estaban dispuestos a tolerar los encuestados para que el gobierno redujese la tasa de contagios en el Perú (Mejia et al., 2021). Se tomó en cuenta el $80 \%$ como valor mínimo que requería cada potencia estadística para poder tener una suficiente consistencia entre los cruces de las variables. No se logró una potencia adecuada para el caso de los estudios técnicos (39\%), para los agnósticos (25\%) y para las religiones: evangélica $(75 \%)$ y agnóstico $(11 \%)$.

La investigación principal generó un proyecto que se sometió al comité de ética de la Universidad Privada Antenor Orrego (Mejia et al., 2021). Para esta investigación se generó otro proyecto, que fue sometido al comité de ética de la Universidad Continental. Para el análisis se descargó la base de datos ya obtenida, esto en una hoja del programa Microsoft Excel 2019, luego esta base fue analizada para la depuración de la misma, aquí es donde se ejecutó 
el control de calidad de los datos. Este control de calidad buscó las exclusiones según los criterios de selección, luego de esto se exportó la base de datos al programa estadístico Stata (versión 11,1 con licencia para funcionamiento obtenida por el estadístico del grupo).

La variable outcome fue la percepción de estar de acuerdo con el "sacrificar a un paciente con COVID-19 grave", y permitió conocer la actitud que tendrían los encuestados frente a un paciente grave infectado por SARS-CoV2. Esta se evaluó mediante la pregunta "Estoy de acuerdo que si alguien tiene un caso grave de coronavirus se le debe sacrificar o dejar morir para evitar el contagio". Esta pregunta tuvo 5 posibles respuestas: Muy de acuerdo, de acuerdo, indiferente, en desacuerdo y muy en desacuerdo. Para el análisis bivariado y multivariado se juntó las categorías muy de acuerdo + de acuerdo (siendo esta la categoría principal) vs las categorías indiferentes + en desacuerdo + muy en desacuerdo (siendo este la categoría de comparación). Esta variable principal se cruzó con las variables del sexo del encuestado (masculino, femenino), la edad que tenía el encuestado al momento de la encuesta (variable tomada en su forma cuantitativa), el grado de instrucción de los encuestados (ninguna, primaria, secundaria, técnico, superior y posgrado) y la orientación religiosa (católica, evangélica, testigo de Jehová, evangélica, mormón agnóstico, ateo y otros).

También se usó las escalas: "KNOW-P-COVID-19", para medir los conocimientos básicos respecto al COVID-19 (Mejia et al., 2020 b); "F-COVID-19", para medir la percepción fatalista ante el posible contagio (Mejia, et $a l ., 2020$ c) y "MED-COVID-19", para medir el miedo o temor ante las noticias de los medios de comunicación (Mejia et al., 2020 d). Se analizó las respuestas de estas en su forma cuantitativa.

Para proceder al análisis de datos primero se procedió a describir la población, esto según quienes estaban o no de acuerdo con el sacrificio de personas, para esto se obtuvo las frecuencias y porcentajes de cada una de las variables categóricas. Además, se obtuvo la mediana y rangos intercuartílicos de la variable cuantitativa (la edad). También se obtuvo los valores p con las pruebas estadísticas del chi cuadrado (para el cruce de las variables categóricas) y la suma de rangos (para el cruce versus la edad). Luego se procedió al análisis bivariado y multivariado de la investigación, en donde se obtuvo los valores de las razones de prevalencia (RP), los intervalos de confianza al 95\% y los valores p de cada cruce. Esto gracias al uso de los modelos lineales generalizados (con la familia Poisson, la función de enlace log y con el uso de modelos para varianzas robustas). Es importante mencionar que cada cruce se consideró como estadísticamente significativo si tenía un valor p menor de 0,05 (esto fue el criterio para pasar del análisis bivariado a multivariado), además, siempre se trabajó con un nivel de confianza al 95\%.

\section{Resultados}

De los 3331 encuestados en el Perú, el 8,1\% (269) estaban muy de acuerdo con que sacrifiquen a las personas con COVID-19 grave, el 8,9\% (297) estuvo de acuerdo, el 15,7\% (524) fue indiferente ante la pregunta, el 20,1\% (670) estuvo en desacuerdo y el 47,2\% (1571) estuvo muy en desacuerdo (Figura 1).

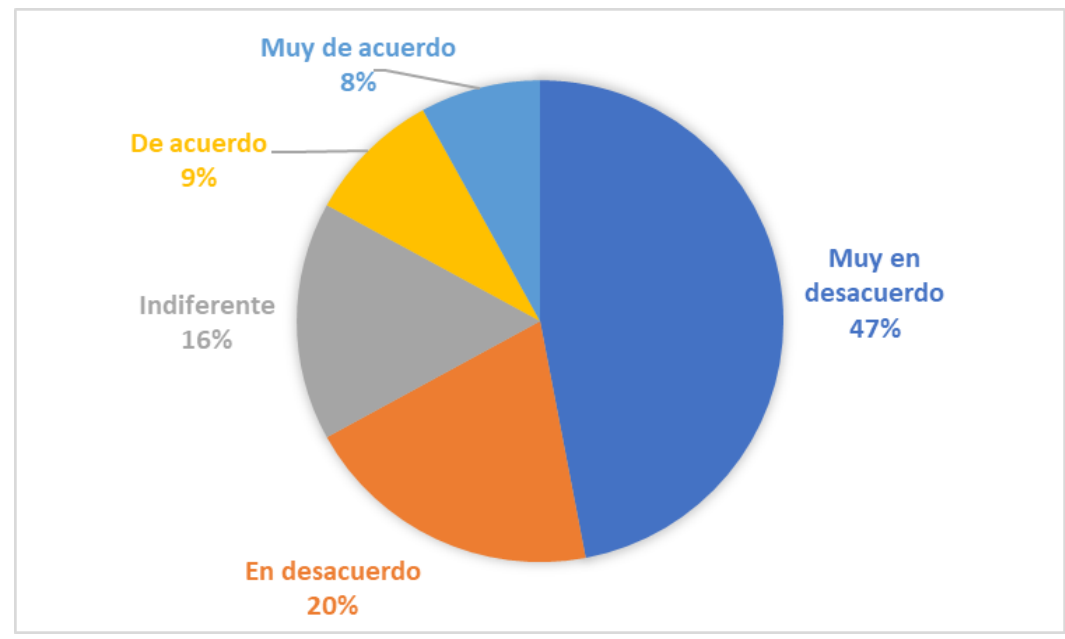

Figura 1. Percepción del poder realizar sacrificio o eutanasia en infectados durante la primera ola del COVID-19 en Perú

Cuando se realizó el análisis descriptivo y bivariado de los factores socio-educativos asociados a la posibilidad de sacrificar a las personas que padecen de COVID-19 grave, se encontró que esto estuvo asociado al sexo (los hombres estaban más de acuerdo con el sacrificar, $\mathrm{p}<0,001)$, también estuvo asociados con el grado de instrucción $(\mathrm{p}=0,002)$, a los puntajes que obtuvieron en los test's de fatalismo $(\mathrm{p}<0,001)$, nivel de conocimientos $(\mathrm{p}<0,001)$ y la percepción de miedo o exageración por parte de los medios de comunicación (p<0,001) (Tabla 1). 
Tabla 1. Análisis descriptivo y bivariado de los factores socio-educativos asociados a la posibilidad de sacrificar a las personas que padecen de COVID-19 grave en el Perú.

\begin{tabular}{|c|c|c|c|}
\hline \multirow{2}{*}{ Variable } & \multicolumn{2}{|c|}{ Sacrificaría a pacientes graves } & \multirow{2}{*}{ Valor $p$} \\
\hline & No & $\mathrm{Si}$ & \\
\hline \multicolumn{4}{|l|}{ Sexo } \\
\hline Masculino & $1171(79,3 \%)$ & $306(20,7 \%)$ & \\
\hline Femenino & $1594(86,0 \%)$ & $260(14,0 \%)$ & $<0,001$ \\
\hline Edad (años)* & $23(20-28)$ & $23(21-28)$ & 0,159 \\
\hline \multicolumn{4}{|l|}{ Instrucción } \\
\hline Ninguna & $10(76,9 \%)$ & $3(23,1 \%)$ & \\
\hline Primaria & $22(71,0 \%)$ & $9(29,0 \%)$ & \\
\hline Secundaria & $432(85,5 \%)$ & $73(14,5 \%)$ & \\
\hline Técnico & $202(75,1 \%)$ & $67(24,9 \%)$ & \\
\hline Universidad & $1882(83,3 \%)$ & $378(16,7 \%)$ & \\
\hline Posgrado & $217(85,8 \%)$ & $36(14,2 \%)$ & 0,002 \\
\hline \multicolumn{4}{|l|}{ Orientación religiosa } \\
\hline Católica & $1862(83,6 \%)$ & $365(16,4 \%)$ & \\
\hline Evangélica & $330(81,1 \%)$ & $77(18,9 \%)$ & \\
\hline Agnóstico & $252(84,3 \%)$ & $47(15,7 \%)$ & \\
\hline Ateo & $125(77,6 \%)$ & $36(22,4 \%)$ & \\
\hline Cristiano & $59(88,1 \%)$ & $8(11,9 \%)$ & \\
\hline Adventista & $39(84,8 \%)$ & $7(15,2 \%)$ & \\
\hline Testigo de Jehová & $4(100,0 \%)$ & $0(0,0 \%)$ & \\
\hline Iglesia de los últimos días & $18(78,3 \%)$ & $5(21,7 \%)$ & \\
\hline Mormón & $13(76,5 \%)$ & $4(23,5 \%)$ & \\
\hline Budista & $9(75,0 \%)$ & $3(25,0 \%)$ & \\
\hline Otra & $54(79,4 \%)$ & $14(20,6 \%)$ & 0,489 \\
\hline Suma de fatalismo* & $20(17-24)$ & $24(20-29)$ & $<0,001$ \\
\hline Suma del conocimiento* & $7(6-8)$ & $6(5-7)$ & $<0,001$ \\
\hline Suma de miedo por comunicación* & $36(29-42)$ & $42(33-48)$ & $<0,001$ \\
\hline
\end{tabular}

En el análisis multivariado de los factores socio-educativos asociados a la posibilidad de sacrificar a las personas que padecen de COVID-19 grave, se encontró que esto estuvo asociado al sexo (las mujeres estaban menos de acuerdo con el sacrificar, RPa: 0,73; IC95\%: 0,62-0,86; p<0,001), también a la orientación religiosa (los ateos estaban más de acuerdo con el sacrificar, RPa: 1,40; IC95\%: 1,16-1,69; p<0,001), a los puntajes que obtuvieron en los test's de fatalismo (RPa: 1,09; IC95\%: 1,07-1,11; p<0,001), nivel de conocimientos (RPa: 0,85; IC95\%: 0,81-0,88; p<0,001) y la percepción de miedo o exageración por parte de los medios de comunicación (RPa: 1,02; IC95\%: 1,01-1,03; p<0,001) (Tabla 2).

Tabla 2. Estadística analítica de los factores socio-educativos asociados a la posibilidad de sacrificar a las personas que padecen de COVID-19 grave en el Perú

\begin{tabular}{|c|c|c|}
\hline \multirow{2}{*}{ Variable } & \multicolumn{2}{|c|}{ Estadística analítica } \\
\hline & Bivariado & Multivariado \\
\hline \multicolumn{3}{|l|}{ Sexo } \\
\hline Masculino & Ref. & Ref. \\
\hline Femenino & $0,68(0,58-0,78)<0,001$ & $0,73(0,62-0,86)<0,001$ \\
\hline Edad (años)* & $1,01(0,99-1,01) 0,152$ & No entró al modelo \\
\hline \multicolumn{3}{|l|}{ Instrucción } \\
\hline Ninguna & Ref. & No entró al modelo \\
\hline Primaria & $1,26(0,60-2,64) 0,544$ & No entró al modelo \\
\hline Secundaria & $0,63(0,32-1,25) 0,182$ & No entró al modelo \\
\hline Técnico & $1,08(0,56-2,08) 0,820$ & No entró al modelo \\
\hline Universidad & $0,72(0,38-1,38) 0,326$ & No entró al modelo \\
\hline Posgrado & $0,62(0,31-1,24) 0,173$ & No entró al modelo \\
\hline \multicolumn{3}{|l|}{ Orientación religiosa } \\
\hline Católica & Ref. & Ref. \\
\hline Evangélica & $1,15(0,93-1,43) 0,193$ & $1,01(0,85-1,21) 0,875$ \\
\hline Agnóstico & $0,96(0,71-1,30) 0,790$ & $1,17(0,86-1,59) 0,331$ \\
\hline Ateo & $1,36(1,04-1,78) 0,023$ & $1,40(1,16-1,69)<0,001$ \\
\hline Cristiano & $0,73(0,39-1,40) 0,325$ & $0,87(0,47-1,61) 0,665$ \\
\hline Adventista & $0,93(0,57-1,51) 0,764$ & $0,75(0,45-1,25) 0,273$ \\
\hline Testigo de Jehová & No converge & No converge \\
\hline Iglesia de los últimos días & $1,33(0,67-2,63) 0,418$ & $1,04(0,65-1,66) 0,883$ \\
\hline Mormón & $1,44(0,55-3,73) 0,458$ & $1,56(0,51-4,75) 0,437$ \\
\hline Budista & $1,53(0,68-3,43) 0,307$ & $2,07(0,85-5,06) 0,109$ \\
\hline Otra & $1,26(0,78-2,01) 0,344$ & $1,31(0,81-2,12) 0,271$ \\
\hline Suma de fatalismo* & $1,12(1,09-1,14)<0,001$ & $1,09(1,07-1,11)<0,001$ \\
\hline Suma del conocimiento* & $0,76(0,73-0,80)<0,001$ & $0,85(0,81-0,88)<0,001$ \\
\hline Suma de miedo por comunicación* & $1,05(1,03-1,06)<0,001$ & $1,02(1,01-1,03)<0,001$ \\
\hline
\end{tabular}




\section{Discusión}

En esta investigación se evidenció que el 17\% de la población encuestada estuvo muy de acuerdo o de acuerdo con sacrificar a los pacientes con COVID-19 grave, en contraste con el $20 \%$ que estuvo en desacuerdo y un $16 \%$ a los que les resulta indiferente sacrificar o no a los pacientes con una presentación complicada del COVID-19. Davis (2020), menciona que algo similar se evidenció en los Estados Unidos de América, dónde se reaperturó muy tempranamente los negocios a pesar de la persistencia del virus, pues las autoridades consideraban que era mejor sacrificar a los adultos mayores, quienes son una población vulnerable en esta pandemia, esto antes que sacrificar la economía del país, y no solo eso, sino que autoridades (como el Gobernador de Texas) consideraban que los adultos mayores lo preferían de esa manera. Por otro lado, en España, uno de los países de la Unión Europea más afectados por esta pandemia, se planteó la opción de brindar la eutanasia asistida como parte del manejo de pacientes con COVID-19 grave, pues en este país la eutanasia está despenalizada (Mahzaniar et al., 2021). Esta situación evidencia que ya sea por una percepción fatalista de la enfermedad, por decisiones políticas o médicas, entre otras muchas que puede haber; algunas personas pueden formular la opción de sacrificar a aquellos pacientes que se contagiaron con este nuevo virus. Nuestra investigación es un primer reporte científico que se da en nuestra región, teniendo solo noticias y declaraciones políticas. Por lo que, esta percepción que tuvieron miles de peruanos (dentro de los que encuestamos) muestra que pudo ser una realidad más extendida. Lamentablemente el tipo de muestreo no puede permitir extrapolar este hallazgo a la totalidad de departamentos o incluso al país entero; pero suponemos que esta idea del "sacrificio" estuvo mucho más extendida en algunos sectores. Lo que debe propiciar más investigación por parte de grupos de investigación en temas sociales.

Asimismo, se evidenció que las opiniones a favor de sacrificar pacientes con COVID-19 grave se asociaron con el sexo, pues existió un mayor porcentaje de encuestados de sexo masculino que estuvieron de acuerdo con sacrificar a este tipo de pacientes (21\%), esto en comparación con las participantes de sexo femenino (14\%). Estos resultados fueron similares a los obtenidos por Vijayalakshmi et al., (2017), quienes encontraron que el 57\% de los enfermeros hombres estaba de acuerdo con interrumpir el apoyo nutricional si el paciente deseaba la eutanasia, siendo estos resultados estadísticamente significativos. De la misma manera, Álvarez Ovelar et al., (2019) reportaron en su estudio que el $57 \%$ de los estudiantes varones estaban dispuestos a practicar la eutanasia, pues la consideraban una muerte digna para ciertos pacientes. Entonces, queda en evidencia que el género juega un papel importante en la decisión de sacrificar o no a los pacientes con enfermedades que tienen un pronóstico desalentador.

Por otro lado, la religión o creencia profesada por los encuestados también se asoció con la percepción respecto al sacrificio de pacientes con COVID-19 grave, evidenciándose que entre los encuestados ateos hubo mayor proporción de opiniones a favor del sacrificio (22\%). Este aspecto es particularmente importante en la manera en cómo los distintos individuos asimilan y perciben los eventos relacionados con el final de la vida. Muchas personas en función de su religión o creencias generan juicios de valor a favor o en contra de finalizar voluntariamente la vida de un ser humano. Por ejemplo, Lee et al., (2017) evidenciaron en un estudio realizado en ciudadanos de Nueva Zelanda que las personas con alguna creencia religiosa se encontraban menos dispuestos a apoyar la despenalización de la eutanasia en su país. De la misma manera, un estudio realizado en estudiantes de medicina evidenció que el $94 \%$ de los estudiantes que eran agnósticos estaban a favor de la eutanasia (Álvarez Ovelar et al., 2019). Por lo cual, todo este constructo dogmático influye en las opiniones de los encuestados ante un tema polémico, como el que se aborda en la presente investigación.

Otro aspecto importante es el papel de los medios de comunicación en la percepción de cada individuo, pues durante el transcurso de la pandemia se ha visto como muchas veces los medios de comunicación comparten información con contenido muy alarmante e incluso sentimental, esto con la finalidad de captar mayor atención de la audiencia (Bunker, 2020). Sin embargo, todo esto influye en la percepción de las personas ante una amenaza constante, como el hecho de enfermarse de COVID-19 y fallecer (Wang et al., 2020). Además, la información tendenciosa emitida por los medios de comunicación, de forma sinérgica con pensamientos religiosos fatalistas, incrementa el riesgo de padecer de síntomas o incluso de trastornos depresivos, los cuales se asocian también a un riesgo incrementado de suicidio o ideas de sacrificio (Mejia et al., 2020 a).

La aplicación de los test de fatalismo y de percepción de miedo o exageración por parte de los medios de comunicación permiten poner en evidencia que, a mayor puntuación en estos test, existe una mayor probabilidad de estar a favor del sacrificio de los pacientes con presentaciones graves de COVID-19. Esto guarda relación con lo reportado por otras investigaciones, donde se demuestra que el enterarse del padecimiento de una enfermedad crónica o con alta letalidad se asociaba a padecer depresión (Hernandez Sampayo et al., 2020). Por otro lado, el padecer COVID19, aunque sea en un paciente sin factores de riesgo o con cuadros leves o moderados de la enfermedad, podría generar temores innecesarios o incluso estigmas sociales, por el simple hecho de estar contagiado con este nuevo coronavirus, y este temor innecesario podría generar repercusiones en la salud física y mental de los pacientes y sus familiares (Valero Cedeño et al., 2020). Es en este contexto donde el estado debe generar un rol rector, ya que, se sabe que muchas veces 
los medios de comunicación transmiten noticias de tipo "sensacionalista", lo que puede acrecentar en demasía la idea de fatalismo o del miedo en la población. Por poner un ejemplo, en el Perú un medio actuó de manera irresponsable, transmitiendo muchas noticias falsas (cómo el hecho de que había un turismo de vacunas en el país de Chile, que la eficacia de las vacunas chinas era menor al 33,3\%, entre otras muchas). Siendo Willax (el canal en mención) un medio común para este tipo de noticias; situación que debería ser regulada por el estado (a sabiendas que los canales deben gozar de independencia editorial, pero también que si transmiten información falsa deberían ser castigados). Este es un claro ejemplo de que la población puede dejarse llevar por las noticias difundidas.

El conocimiento de los participantes se asoció también con las diferentes opiniones frente al fenómeno estudiado; ya que, ante un mayor conocimiento básico de la enfermedad por parte de los participantes estuvieron menos de acuerdo con sacrificar a los pacientes. Esto también se ha visto en otros estudios (Lee et al., 2017), pudiendo deberse al hecho que el conocimiento genera que se tenga información menos tendenciosa y con mayor respaldo científico en lo correspondiente a esta enfermedad. Un estudio multicéntrico, realizado en 26 países, encontró que fueron las personas con niveles más bajos de educación formal quienes mostraron niveles más altos de estrés (Kowal et al., 2020). También Elsharkawy \& Abdelaziz, (2020), reportaron que las personas más jóvenes, así como, los estudiantes universitarios de los primeros años los que tenían menores niveles de conocimientos acerca de la COVID-19, sin embargo, tenían niveles más altos de miedo e incertidumbre. Por lo cual, es importante que se siga informando a la población acerca esta nueva enfermedad y así se logre disminuir la brecha de conocimiento que existe, la cual podría llevar a la población a tomar o pensar en decisiones drásticas.

En este estudio se tuvo la limitación del no poder extrapolar los resultados a la totalidad de la población peruana, esto debido a que no se realizó un muestreo aleatorio, ni por ciudades, ni por departamentos, ni de otro modo que permitiese la extrapolación. Esto principalmente debido al hecho que en el momento que se realizó la encuesta el Perú atravesaba las primeras semanas de inmovilización social, cuarentenas rígidas y todas las medidas restrictivas; por lo que, no se pudo tener la posibilidad de obtener listas y con ello la aleatorización. Sin embargo, esta es una limitación relativa, ya que, se obtuvo una muestra de más de 3000 peruanos en los departamentos más representativos, lo que nos puede mostrar las asociaciones más importantes que se obtuvieron para la pregunta de sacrificio ante otros factores asociados (solo en cuatro cruces no se tuvo una potencia adecuada, por lo que, en esos cruces se deben manejar con cuidado). Se espera que futuras investigaciones del área de salud mental muestren los factores que podrían estar influyendo en esta asociación, ya que, el que una población manifieste en un porcentaje importante que estaría a favor del sacrifico de enfermos de una cierta patología muestra que existe un miedo, conocimientos inadecuados u otros que generen esta respuesta, futuras investigaciones tendrán que medir esto en un mayor contexto (con una mayor población y con un diseño que busque causalidad), así como, evaluar otras preguntas que explicarían de mejor forma esta realidad.

Por todo lo antes mencionado, se concluye que uno de cada seis peruanos estuvo de acuerdo o muy de acuerdo con que se sacrifique a personas que tenían COVID-19 grave. Además, se obtuvo una asociación de esta percepción con el que sea del sexo masculino y con los ateos, ambos tuvieron mayor aceptación por el sacrificio, así como, los que tuvieron mayor porcentaje en el test de fatalismo, los que tuvieron menor puntaje en el test de conocimiento y los que tuvieron un mayor puntaje en el test de la percepción de miedo o exageración que transmitían los medios de comunicación.

\section{Conflicto de intereses}

Los autores declaran no tener conflicto de interés.

\section{Agradecimientos}

Agradecemos al grupo COVID-19-GIS-Peru, que cedió, la base de datos primaria que se utilizó para la obtención de los resultados de esta investigación. Además, sus miembros ayudaron en la recolección de la data primaria. Además, al estudiante universitario Jean Jurik Vera Gonzales, que dio la idea para la pregunta principal de la investigación.

\section{Referencias}

Abdoli, A. (2020). Iran, sanctions, and the COVID-19 crisis. Journal of Medical Economics, 23:12:1461-1465, https://doi.org/10.1080/13696998.2020.1856855

Alderete-Aguilar, C., Cruz-Maycott, R., Candela-Iglesias, M., Rodríguez Estrada, E., \& Reyes-Terán, G. (2017). Assessment of depression, anxiety, hopelessness and suicidal risk in HIV+ inpatients. Salud mental (Mexico City, Mexico), 40(1):23-28. https://doi.org/10.17711/SM.0185-3325.2017.004

Álvarez Ovelar, R. D., Martínez Román, M. D., Rodas Benítez, K. Y., \& Invernizzi Prats, J. M. (2019). Percepción sobre la Eutanasia en Estudiantes de Medicina de dos Universidades del Paraguay. 2017. Revista de 
Investigación

Científica

https://doi.org/10.36003/Rev.investig.cient.tecnol.V3N1(2019)2

Tecnológica,

$3(1): 21-31$.

Asmundson, G., \& Taylor, S. (2020). How health anxiety influences responses to viral outbreaks like COVID-19: What all decision-makers, health authorities, and health care professionals need to know. Journal of anxiety disorders, 71:102211. https://doi.org/10.1016/j.janxdis.2020.102211

Bunker D. (2020). ¿En quién confías? La destrucción digital de la conciencia situacional compartida y la infodemia COVID-19. Revista internacional de gestión de la información, 55:102201. https://doi.org/10.1016/j.ijinfomgt.2020.102201

Carrieri, M. P., Marcellin, F., Fressard, L., Préau, M., Sagaon-Teyssier, L., Suzan-Monti, M., Guagliardo, V., Mora, M., Roux, P., Dray-Spira, R., Spire, B., \& ANRS-VESPA2 Study Group. (2017). Suicide risk in a representative sample of people receiving HIV care: Time to target most-at-risk populations (ANRS VESPA2 French national survey). PloS One, 12(2):e0171645. https://doi.org/10.1371/journal.pone.0171645

Davis, S. P. (2020). Euthanasia of the Coronavirus - COVID-19. Online journal of health ethics, 16(1):1-4. https://doi.org/10.18785/ojhe.1601.02

Du, L., Shi, H.-Y., Yu, H.-R., Liu, X.-M., Jin, X.-H., Yan-Qian, Fu, X.-L., Song, Y.-P., Cai, J.-Y. \& Chen, H.-L. (2020). Incidence of suicide death in patients with cancer: A systematic review and meta-analysis. Journal of Affective Disorders, 276: 711-719.

Dubón-Peniche, M. D. C., \& Bustamante-Leija, L. E. (2020). Between illness and death: "Euthanasia". Cirugia y Cirujanos, 88(4):519-525. https://doi.org/10.24875/ciru.18000626

Elsharkawy, N. B., \& Abdelaziz, E. M. (2020). Levels of fear and uncertainty regarding the spread of coronavirus disease (COVID-19) among university students. Perspectives in Psychiatric Care, ppc.12698. https://doi.org/10.1111/ppc.12698

Hernandez Sampayo, L., Sterling Diaz, K., \& Potosi Arevalo, A. (2020). Situaciones emocionales previas al diagnóstico de cáncer de mama. Paradigmas Socio-Humanísticos, 2(1):10-16. https://orcid.org/0000-0002-7031-9266

Infobae. (2020, noviembre 4). Corea del Norte confina a sus enfermos de COVID-19 en "campos de cuarentena" y los deja morir. Infobae Noticias. Disponible en: https://www.infobae.com/america/mundo/2020/11/04/corea-delnorte-confina-a-sus-enfermos-de-covid-19-en-campos-de-cuarentena-y-los-deja-morir/ (Acceso marzo 2021)

Kowal, M., Coll-Martín, T., Ikizer, G., Rasmussen, J., Eichel, K., Studzińska, A., Koszałkowska, K., Karwowski, M., Najmussaqib, A., Pankowski, D ., Lieberoth, A. \& Ahmed, O. (2020). ¿Quién está más estresado durante la pandemia de COVID-19? Datos de 26 países y áreas. Psicología Aplicada. Salud y bienestar, 12 (4):946966. https://doi.org/10.1111/aphw.12234

Lee, C. H., Duck, I. M., \& Sibley, C. G. (2017). Demographic and psychological correlates of New Zealanders support for euthanasia. The New Zealand Medical Journal, 130(1448):9-17. Diaponible en: https://assets-global.websitefiles.com/5e332a62c703f653182faf47/5e332a62c703f6fbf92fcffe Lee\%20FINAL.pdf (Acceso marzo 2021)

Mahzaniar, M., Lubis, M. R., Nasution, A., \& Maryani, H. (2021). Euthanasia for COVID-19 patients during the pandemic. European Journal of Social Sciences Studies, 6(4):296-303. http://dx.doi.org/10.46827/ejsss.v6i4.1087

Mejia CR, Ticona D, Rodriguez-Alarcon JF, Campos-Urbina AM, et al. (2021). Percepción de las medidas de salud pública en Perú para frenar el avance de la COVID-19. Revista Cubana de Investigaciones Biomédicas., 40(1):e737. Disponible en: http://scielo.sld.cu/scielo.php?script=sci_arttext\&pid=S0864-03002021000100005 (Acceso marzo 2021)

Mejia, C. R., Quispe-Sancho, A., Rodriguez-Alarcon, J. F., Ccasa-Valero, L., Ponce-López, V. L., Varela-Villanueva, E. S., Marticorena-Flores, R. K., Chamorro-Espinoza, S. E., Avalos-Reyes, M. S., \& Vera-Gonzales, J. J. (2020 a). Factores asociados al fatalismo ante la COVID-19 en 20 ciudades del Perú en marzo 2020. Revista Habanera de Ciencias Médicas, 19(2), 3233.

Mejia, C. R., Rodríguez-Alarcón, J. F., Carbajal, M., Pérez-Espinoza, P., Porras-Carhuamaca, L. A., Sifuentes-Rosales, J., Contreras-Cabrera, J. M., Carranza-Esteban, R. F., \& Ruiz-Mamani, P. G. (2020 b). Fatalismo ante la posibilidad de contagio por el coronavirus: Generación y validación de un instrumento (F-COVID-19). Kasmera, 48(1):1-10.https://doi.org/10.5281/zenodo.3732353

Mejia, C. R., Rodriguez-Alarcon, J. F., Carbajal, M., Sifuentes-Rosales, J., Campos-Urbina, A. M., Charri, J. C., GarayRios, L., Al-Kassab-Cordova, A., Mamani-Benito, O., \& Apaza-Tarqui, E. E. (2020 c). Validación de una escala 
breve para la medición del nivel de conocimientos básicos acerca del Coronavirus, Perú (KNOW-P-COVID-19). Kasmera, 48(1):1-10. https://doi.org/10.5281/zenodo.3827988

Mejia, C. R., Tovani-Palone, M. R., Ticona, D., Rodriguez-Alarcon, J. F., Campos-Urbina, A. M., Catay-Medina, J. B., Porta-Quinto, T., Garayar-Peceros, H., Ignacio-Quinte, C., Carranza Esteban, R. F., \& Ruiz Mamani, P. G. (2020 d). The media and their informative role in the face of the Coronavirus disease 2019 (COVID-19): Validation of fear perception and magnitude of the issue (MED-COVID-19). Electronic Journal of General Medicine, 17(6):em239. https://doi.org/10.29333/ejgm/7946

Pirkis, J., John, A., Shin, S., DelPozo-Banos, M., Arya, V., Analuisa-Aguilar, P., Appleby, L., Arensman, E., Bantjes, J., Baran, A., Bertolote, J. M., Borges, G., Brečić, P., Caine, E., Castelpietra, G., Chang, S.-S., Colchester, D., Crompton, D., Curkovic, M., ... Spittal, M. J. (2021). Suicide trends in the early months of the COVID-19 pandemic: an interrupted time-series analysis of preliminary data from 21 countries. The Lancet. Psychiatry, 8(7):579-588. https://doi.org/10.1016/S2215-0366(21)00091-2

Rodriguez Casas, R. C. (2001). Eutanasia: aspectos éticos controversiales. Revista medica herediana, 12(1):32-36. Disponible en: http://www.scielo.org.pe/scielo.php?script=sci_arttext\&pid=S1018-130X2001000100007 (Acceso marzo 2021)

Torres Lio-Coo, V., García Gutierrez, E., Casamayor Laime, Z., Torres Ruiz, J. R., Güidi Virues, E., \& Abreu García, M. D. (2009). Consideraciones sobre la eutanasia como práctica médica. Revista cubana de medicina militar, 38(2):1-9. Disponible en: http://scielo.sld.cu/scielo.php?script=sci_arttext\&pid=S0138$\underline{65572009000200006}$ (Acceso marzo 2021)

Valero Cedeño, N. J., Vélez Cuenca, M. F., Duran Mojica, Á. A., \& Torres Portillo, M. (2020). Afrontamiento del COVID-19: estrés, miedo, ansiedad y depresión. Enfermeria Investiga, 5(3):63-70. Disponible en: https://revistas.uta.edu.ec/erevista/index.php/enfi/article/view/91 (Acceso marzo 2021)

Vijayalakshmi, P., Nagarajaiah, Reddy, P. D., \& Suresh, B. M. (2018). Indian Nurses' Attitudes Toward Euthanasia: Gender Differences. Omega, 78(2):143-160. https://doi.org/10.1177/0030222816688576

Wang, C., Pan, R., Wan, X., Tan, Y., Xu, L., Ho, C. S., \& Ho, R. C. (2020). Immediate Psychological Responses and Associated Factors during the Initial Stage of the 2019 Coronavirus Disease (COVID-19) Epidemic among the General Population in China. International journal of environmental research and public health, 17(5):1729. https://doi.org/10.3390/ijerph17051729

White, M. D., \& Fradella, H. F. (2020). Vigilancia de una pandemia: órdenes de quedarse en casa y lo que significan para la policía. Revista estadounidense de justicia penal: AJCJ :1-16. https://doi.org/10.1007/s12103-020$\underline{09538-0}$ 